\title{
Electron correlation in double ionization of excited helium by fast ion impact
}

\author{
L G Gerchikov†, S A Sheinerman $\ddagger$, M Schulz $\uparrow$, \\ R Moshammer§, and J Ullrich
}

$\dagger$ Department of Experimental Physics, St.-Petersburg State Technical University, St.-Petersburg 195251, Russia

† Department of Physics, St.-Petersburg State Maritime Technical University, St.-Petersburg 198262, Russia

ๆ Physics Department and Laboratory for Atomic, Molecular and Optical Research, University of Missouri-Rolla, Missouri 65409, USA

$\S$ Max-Planck-Institut für Kernphysik, Saupfercheckweg 1, D-69117, Heidelberg, Germany

PACS numbers: $34.50 \mathrm{Fa}, 31.25 \mathrm{Jf}$

\begin{abstract}
.
The correlation function between two emitted electrons has been calculated as a function of their momentum difference for double ionization of the 1s $2 \mathrm{~s}$ excited He atoms by fast ion impact. A simple quantum mechanical approach is developed to take into account the dynamics of two-electron ejection as well as the effects of electron exchange and correlated electron motion in the final state. A clear difference between the correlation functions of excited and ground state targets is reported.
\end{abstract}




\section{Introduction}

The role of electron correlation in ion - atom collisions has been extensively studied, experimentally as well as theoretically for more than two decades (see e.g. McGuire 1997) mainly on the basis of total cross section mesuraments. Due to the recent development of multi-electron - ion-momentum spectrometers with large momentum acceptance (Dörner et al 2000) differential measurements have become feasible (see e.g. Bapat et al 1999, Bapat et al 2000, Keller et al 1997) and, moreover, intensity interferometry methods (Schulz et al 2000) are applicable for the first time to study electron correlation in double ionization processes. Within this method two kinds of intensity distributions, $I_{\text {cor }}$ and $I_{\text {uncor }}$, for double ionization are determined. The first intensity, $I_{c o r}$, refers to the probability distribution of finding two electrons with a certain momentum difference $\Delta p=\left|\mathbf{p}_{1}-\mathbf{p}_{2}\right|$ after double ionization of a single atom, while $I_{\text {uncor }}$ is the emission intensity at identical $\Delta p$ taking two completely uncorrelated electrons from different ionizing collision events (so-called event mixing mode, see also Boal et al 1990). Their ratio, the so called correlation function $R(\Delta p)=I_{\text {cor }}(\Delta p) / I_{\text {uncor }}(\Delta p)-1$, characterizes to what extent two emitted electrons are correlated. For two completely independent electrons $R=0$, correlation favoring for the electron emission at certain momentum difference $R>0$ and $R<0$ for those $\Delta p$ where the electron emission is suppressed. According to its definition the correlation function $R(\Delta p)$ extracts from the experimental data information concerning the relative correlated motion of two emitted electrons.

So far both the experimental as well as theoretical investigation of correlation functions (Schulz et al 2000, Feuerstein et al 2001, Schulz et al 2001, Gerchikov and Sheinerman 2001) have been carried out for ground state targets. According to the experimental study of Schulz et al (2000) the correlation function is, as expected, largely determined by electron-electron correlation effects, but it was found to be essentially unaffected by the collision dynamics. Thus, measurements of $R$ have revealed close results for projectiles with different charges and velocities, i.e. at very different perturbations. Furthermore, our previous study of double ionization from the ground state shows that the correlation function is also quite insensitive to the choice of the target atom. All together, it was finally concluded that the observed features in the data are dominated by electron-electron correlation in the final continuum state rather than in the initial state (Schulz et al 2000, Gerchikov and Sheinerman 2001). The main force that governs the relative motion of two escaping electrons is their Coulomb interaction. Two other contributions to the correlation function, the exchange processes between the two emitted electrons and the electron correlation in the initial state, seem to be less important.

As was suggested by Schulz et al 2001 the initial state could affect the correlation function by defining the initial separation for the correlated motion of the two electrons during the ionization. The characteristic initial interelectron distance is determined by the size of the target atom. In our previous work we used $H e$ and $N e$ target atoms in 
the ground state whose sizes are actually very close. Therefore, it is quite natural that a similar behavior of the correlation functions is revealed. Another shape of $R$ function can be expected, however, in the case of an excited target whose size is much larger than the size of the atomic ground state. Indeed, in this case escaping electrons will start their correlated motion from a larger initial separation.

The influence of other contributions to the correlation function should also be different in the case of an initial excited state. In the excited atom electrons may occupy non-equivalent states and, consequently, the initial wave function of the two emitted electrons can have a different symmetry depending on the total electron spin. This changes the interference between ionization amplitudes originating from an exchange of escaping electrons. On the other hand the fact that two electrons populate quite different electronic states leads to a suppression of the interference effect in comparison with the case of an initial ground state where both target electrons are identical. The considerable difference between the electron levels which are occupied in the excited target will also reduce the correlation in the initial state.

Thus, the study of double ionization of excited states can give valuable additional information about electron correlation in multi-ionization processes, improve our understanding of the nature of different contributions to the correlation function and their relative importance. However, as far as we know, such investigations have not been carried out so far. In order to guide future experiments, we consider the 1s2s states of the helium atom as a simple example of an excited target. This two-electron system can initially be in the ${ }^{1} S$ singlet or ${ }^{3} S$ triplet state. We have calculated the correlation function taking into account electron-electron interaction in the final continuum state and exchange between the two ionized electrons. We do not include into our treatment electron correlation in the initial state. This contribution to the correlation function of the ground state was found to be not very essential (Gerchikov and Sheinerman 2001). So we expect it to be small also for the excited states where the two electrons are well separated in both space and energy.

Similarly to our previous work (Gerchikov and Sheinerman 2001) where we considered double ionization of the helium ground state we restrict ourselves to the case of fast ion-atom collisions with small Born parameter. It allows us to describe the interaction of an incoming ion with the target atom in the Born approximation which simplifies considerably the calculation of the ionization cross section. We will demonstrate that in this case the double ionization of the excited target atom nearly exclusively occurs via knock-out of the inner 1s electron and shake-off of the outer 2 s electron. Moreover, because of the high velocity of the projectile, typically impact parameters much larger than the target size are essential for the ionization process. This fact allows us to calculate the ionization cross section in the dipole approximation (see, for example, Berakdar and Klar 1993). It should be noticed that from the technical point of view a calculation of double ionization cross sections for excited atoms turns out to be much more complicated than in the case of initial ground state. In the latter case the relatively small size of the initial target state allowed us to introduce some simplifications 
for the continuum two-electron wave function (Gerchikov and Sheinerman 2001) which made it possible to derive an analytical closed expression for the ionization cross section. Unfortunately, in the case of the excited 1s2s state of helium, these simplifications can not be applied due to the large radius of the outer 2 s electron orbit and time consuming numerical calculations are necessary. However, these calculations can be simplified if we take into account that the radius of the inner 1s electron orbit is much smaller than the radius of the outer $2 \mathrm{~s}$ electron state.

Using these approximations we have calculated the correlation function for the considered process, analyzed its behavior and studied the role of different contributions to electron correlation. Our investigation shows that electron correlation in double ionization of excited states is mainly caused by the interaction of two ionized electrons in the final state. The role of the exchange process between the knock-out and shakeoff electrons as well as the correlation between unequal electrons in the initial state turn out to be considerably less important compared to our calculations for initial ground state (about the role of the initial state correlation see, also, Kheifets et al 2000). The increase in size of the excited atom in comparison with the ground state causes considerable alterations in the behavior of the correlation function. The obtained difference between correlation functions of excited and ground state targets is larger than the achievable resolution of intensity interferometry methods. Therefore, we hope that future experimental studies could verify our theoretical predictions. Furthermore, the observed dependence of the correlation function upon the initial target state might make it possible to use intensity interferometry methods as an effective tool for the diagnostics of atomic and molecular states.

The paper is organized as follows. In section 2 the basic definitions are given together with a detailed description of the evaluation of the double ionization cross section. In section 3 the results of the present calculation, comparison with the corresponding data for the initial ground state targets, analysis and discussion of the different contributions to electron correlation are presented. The atomic system of units $|e|=m_{e}=\hbar=1$ is used throughout.

\section{Method of calculation of the correlation function}

Let us consider double ionization of the excited helium atom in a collision with the fast multi-charged ion $A$ :

$$
A+H e^{*}(1 s 2 s) \rightarrow A+H e^{2+}+e\left(\mathbf{p}_{1}\right)+e\left(\mathbf{p}_{2}\right),
$$

where vectors $\mathbf{p}_{1}$ and $\mathbf{p}_{2}$ denote the momenta of the two escaping electrons. The probability distribution $\Phi\left(\mathbf{p}_{1}, \mathbf{p}_{2}\right)$ for the emission of two electrons with momenta $\mathbf{p}_{1}$ and $\mathbf{p}_{2}$ can be expressed via the differential cross section of this process $d^{6} \sigma / d \mathbf{p}_{1} d \mathbf{p}_{2}$ :

$$
\Phi\left(\mathbf{p}_{1}, \mathbf{p}_{2}\right)=\frac{1}{\sigma_{t o t}} \frac{d^{6} \sigma}{d \mathbf{p}_{1} d \mathbf{p}_{2}}
$$


where $\sigma_{t o t}$ is the total cross section for double ionization

$$
\sigma_{t o t}=\int \frac{d^{6} \sigma}{d \mathbf{p}_{1} d \mathbf{p}_{2}} d \mathbf{p}_{1} d \mathbf{p}_{2}
$$

We are interested in the correlation function $R$ which is determined in accordance with (Schulz et al 2000 and references therein) the experimentally measured intensities: $I_{c o r}(\Delta p)$ of double electron emission in the same ionization event and $I_{\text {uncor }}(\Delta p)$ of two uncorrelated electrons ejected in two independent ionization events as a function of momentum difference of the emitted electrons $\Delta p=\left|\mathbf{p}_{1}-\mathbf{p}_{2}\right|$ :

$$
R(\Delta p)=\frac{I_{\text {cor }}(\Delta p)}{I_{\text {uncor }}(\Delta p)}-1
$$

Intensities $I_{\text {cor }}$ and $I_{\text {uncor }}$ which are meant as a probability distributions can be calculated with the help of the probability distribution for double ionization (2) by integration over the ejected electron momenta with a fixed momentum difference $\Delta p$ :

$$
\begin{aligned}
& I_{\text {cor }}(\Delta p)=(\Delta p)^{2} \int \Phi(\mathbf{p}, \mathbf{p}+\Delta \mathbf{p}) d \mathbf{p} d \Omega_{\Delta \mathbf{p}} \\
& I_{\text {uncor }}(\Delta p)=(\Delta p)^{2} \int G(\mathbf{p}+\Delta \mathbf{p}) G(\mathbf{p}) d \mathbf{p} d \Omega_{\Delta \mathbf{p}},
\end{aligned}
$$

where $G(\mathbf{p})$ is the probability distribution for momentum of a single electron emitted in the double ionization

$$
G(\mathbf{p})=\int \Phi\left(\mathbf{p}, \mathbf{p}^{\prime}\right) d \mathbf{p}^{\prime}
$$

We will consider the case of sufficiently fast ions in which the Born parameter is much smaller than unity, $Q / v \ll 1$, where $Q$ and $v$ are the projectile charge and velocity, respectively. Then, we can describe the interaction between the fast heavy ion of mass $M$ and the target atom in the first Born approximation. In the framework of this approximation, the wave functions of the incoming and outgoing ion with momenta $\mathbf{P}$ and $\mathbf{P}^{\prime}$, respectively, are given by plane waves and the differential cross section is

$$
\frac{d^{6} \sigma}{d \mathbf{p}_{1} d \mathbf{p}_{2}}=\int \frac{M^{2} P^{\prime}}{4 \pi^{2} P}\left|T_{f i}\right|^{2} d \Omega_{\mathbf{P}^{\prime}}
$$

where $T_{f i}$ is the double ionization transition amplitude:

$$
T_{f i}=\left\langle\Psi_{\mathbf{p}_{1} \mathbf{p}_{2}}\left(\mathbf{r}_{a}, \mathbf{r}_{b}\right) \cdot e^{i \mathbf{P}^{\prime} \mathbf{r}}\left|\frac{2 Q}{r}-\frac{Q}{\left|\mathbf{r}-\mathbf{r}_{a}\right|}-\frac{Q}{\left|\mathbf{r}-\mathbf{r}_{b}\right|}\right| \Psi_{a t}^{(e x)}\left(\mathbf{r}_{a}, \mathbf{r}_{b}\right) \cdot e^{i \mathbf{P r}}\right\rangle .
$$

Here $\mathbf{r}, \mathbf{r}_{a}$ and $\mathbf{r}_{b}$ are the positions of the projectile ion and the two target electrons, respectively; two electron wave functions $\Psi_{\mathbf{p}_{1} \mathbf{p}_{2}}$ and $\Psi_{a t}^{(e x)}$ describe the final and initial state of the target electrons. The transition amplitude $T_{f i}$ contains the interaction of the projectile ion with the nucleus of the target atom and its two electrons.

The further evaluation of the ionization amplitude (9) will be performed within the dipole approximation (see, for example, Berakdar and Klar 1993). This approximation 
can be applied because in this paper we will consider the actual experimental situation when the projectile velocity is rather high, $v \gg Q>1$, while the momenta of the detected electrons are restricted. Therefore, the minimum value $q_{\text {min }}$ of the transferred momentum $\mathbf{q}=\mathbf{P}-\mathbf{P}^{\prime}$ is small. Indeed, the minimum momentum transfer, $q_{\text {min }}$, is determined by the ratio of the transferred energy $\Delta E$ and the ion velocity $v$ :

$$
q_{\text {min }}=\frac{\Delta E}{v}=\frac{1}{v}\left(p_{1}^{2} / 2+p_{2}^{2} / 2+E^{2+}\right),
$$

where $E^{2+}$ is the total binding energy of both electrons in the target atom. Due to the high velocity of the projectile ion, $q_{\min }$ turns out to be much smaller than the characteristic momenta of atomic electrons $q_{a t} \sim 1$. On the other hand, the differential cross section (8) decreases rapidly with the increase of $q$. Therefore, the most important transferred momenta $q$ belong to the region $q_{\text {min }}<q<<q_{a t}$ corresponding to processes occurring at impact parameters $\rho$ larger than the size of the target atom, $\rho \sim r \gg r_{a}, r_{b}$. Under these circumstances the dipole approximation can be applied to calculate the ionization cross section. In this case the differential cross section can be presented as:

$$
\frac{d^{6} \sigma}{d \mathbf{p}_{1} d \mathbf{p}_{2}}=\int \frac{q d q d \varphi_{q}}{4 \pi^{2} v^{2}}\left|T_{f i}\right|^{2},
$$

where the ionization amplitude is given by

$$
T_{f i}=\frac{4 \pi Q}{q^{2}} \int d \mathbf{r}_{a} d \mathbf{r}_{b} \Psi_{\mathbf{p}_{1} \mathbf{p}_{2}}^{*}\left(\mathbf{r}_{a}, \mathbf{r}_{b}\right)\left[\mathbf{q}\left(\mathbf{r}_{a}+\mathbf{r}_{b}\right)\right] \Psi_{a t}^{(e x)}\left(\mathbf{r}_{a}, \mathbf{r}_{b}\right),
$$

and $\varphi_{q}$ is the azimuthal angle of the vector $\mathbf{q}$ with respect to the direction of the incoming ion.

In order to calculate the matrix element (12) we need to define the two-electron wave functions of the initial and final states. For the initial 1s2s excited state of the He atom we use the Hartree-Fock (HF) wave function which is written as the antisymmetrized product of the single particle $1 s$ and $2 s \mathrm{HF}$ wave functions $\varphi_{1 s}$ and $\varphi_{2 s}$ :

$$
\Psi_{a t}^{(e x)}\left(\mathbf{r}_{a}, \mathbf{r}_{b}\right)=\frac{1}{\sqrt{2}}\left[\varphi_{1 s}\left(\mathbf{r}_{a}\right) \cdot \varphi_{2 s}\left(\mathbf{r}_{b}\right)+(-1)^{S} \varphi_{1 s}\left(\mathbf{r}_{b}\right) \cdot \varphi_{2 s}\left(\mathbf{r}_{a}\right)\right]
$$

where $S$ is the total spin of two-electron system which is equal to $S=0$ for a singlet initial state and $S=1$ for a triplet initial state. Single electron wave functions $\varphi$ describe the electron motion in the HF self-consistent mean-field potential of the target atom but do not take into account electron correlation caused by the residual interaction. Thus, using HF wave functions (13) we neglect electron correlation in the initial state.

The HF single particle wave functions can be approximated by exponential functions suitable for the following calculations:

$$
\varphi_{v}(r)=\sum_{i=1}^{n} B_{i}^{v} \exp \left(-\beta_{i}^{v} r\right)
$$

where $v=1 s, 2 s ; n=2$ or 3 for $v=1 s$ or $2 s$, respectively; $B_{i}^{v}$ and $\beta_{i}^{v}$ are given in Table 1. The accuracy criterion of such an approximation of HF wave functions proves to be 
better than $\chi^{2} \leq 10^{-8}$. Note, that the single particle $\mathrm{HF}$ wave functions $\varphi_{v}(r)(14)$ are different for the singlet and triplet states. Mainly it concerns the outer, $2 \mathrm{~s}$, electron shell which is less tightly bound in the case of ${ }^{1} S$ helium than in ${ }^{3} S$ helium resulting in a larger radius of the $2 \mathrm{~s}$ orbit for the singlet compared to the triplet state (see Table 1 ).

For the final state of two emitted electrons we take the correlated double continuum BBK wave function with correct asymptotic behavior. Such a wave function has been used by a number of authors for the description of atomic ionization processes (see, for example, Garibotti and Miraglia 1980, Brauner et al 1989). Note, though having the correct asymptotic behavior, the BBK function provides only an approximate description of the real two-electron continuum wave function inside the target atom. Nevertheless, the use of the BBK function in the case of ground state $H e$ provided a very good agreement of the calculated correlation function with experimental data (Gerchkov and Sheinerman 2001). The result is connected with the fact that calculations, based on the use of the BBK wave function, give a good description of the momentum distribution of electrons ejected in double ionization process (Maulbetsch and Briggs 1993, 1994), though such a calculations do not necessarily yield the correct absolute values of the cross sections (McGuire 1997, Lucey et al 1998, Briggs and Schmidt 2000). However, the correlation function defined by (4) depends upon the momentum distributions and is not sensitive to the absolute values of the cross sections. The BBK wave function is a product of two Coulomb waves, $\varphi_{\mathbf{p}_{\mathbf{i}}}\left(\mathbf{r}_{a, b}\right)$, describing the electron motion in the field of the doubly charged nucleus and the factor $F_{\text {int }}\left(\mathbf{p}_{12}, \mathbf{r}_{a b}\right)$ which takes into account the interaction between escaping electrons. This factor is approximated by the product of a so-called Sommerfeld factor $F_{S o m}$ and a confluent hypergeometric function

$$
F_{\text {int }}\left(\mathbf{p}_{12}, \mathbf{r}_{a b}\right)=F_{S o m}\left(\xi_{12}\right) \cdot{ }_{1} F_{1}\left(\mathrm{i} \xi_{12}, 1,-\frac{\mathrm{i}}{2}\left(p_{12} r_{a b}+\mathbf{p}_{12} \mathbf{r}_{a b}\right)\right)
$$

where $\mathbf{r}_{a b}=\mathbf{r}_{a}-\mathbf{r}_{b}$ and $\mathbf{p}_{12}=\mathbf{p}_{1}-\mathbf{p}_{2}$. The Sommerfeld factor $F_{\text {Som }}\left(\xi_{12}\right)$ is equal to

$$
F_{\text {Som }}\left(\xi_{12}\right)=\Gamma\left(1-\mathrm{i} \xi_{12}\right) \exp \left(-\pi \xi_{12} / 2\right) \text { and } \xi_{12}=1 /\left|\mathbf{p}_{1}-\mathbf{p}_{2}\right| \text {, }
$$

where $\Gamma(x)$ is the Gamma function. The antisymmetrized two-electron wave function of the final state is written as

$$
\begin{aligned}
\Psi_{\mathbf{p}_{1} \mathbf{p}_{2}}\left(\mathbf{r}_{a}, \mathbf{r}_{b}\right)=\frac{1}{\sqrt{2}} & {\left[F_{i n t}\left(\mathbf{p}_{12}, \mathbf{r}_{a b}\right) \varphi_{\mathbf{p}_{1}}\left(\mathbf{r}_{a}\right) \cdot \varphi_{\mathbf{p}_{2}}\left(\mathbf{r}_{b}\right)+\right.} \\
& \left.+(-1)^{S} F_{i n t}\left(\mathbf{p}_{12}, \mathbf{r}_{b a}\right) \varphi_{\mathbf{p}_{1}}\left(\mathbf{r}_{b}\right) \cdot \varphi_{\mathbf{p}_{2}}\left(\mathbf{r}_{a}\right)\right],
\end{aligned}
$$

where $S$ is the total spin of two emitted electrons, and the single electron wave functions $\varphi_{\mathbf{p}_{i}}$ are equal to the Coulomb waves in the field of the doubly charged nucleus:

$$
\varphi_{\mathbf{p}_{i}}(\mathbf{r})=(2 \pi)^{-3 / 2} \cdot F_{\text {Som }}\left(\xi_{i}\right) \cdot{ }_{1} F_{1}\left(\mathrm{i} \xi_{i}, 1,-\mathrm{i}\left(p_{i} r+\mathbf{p}_{i} \mathbf{r}\right)\right) \cdot \exp \left(\mathrm{ip}_{i} \mathbf{r}\right),
$$

where $F_{\text {Som }}$ is given by Eq. (16) with $\xi_{i}=-2 / p_{i}$.

It is essential for the further evaluation of the ionization amplitude (12) that the average radius of the $2 \mathrm{~s}$ electron orbit is much larger than the radius of the $1 \mathrm{~s}$ orbit 
$r_{2 s} \gg r_{1 s}$. The 1s electron is located at an average distance $r_{1 s} \sim 1 a . u$. from the nucleus whereas the $2 \mathrm{~s}$ electron is located at the larger distance $r_{2 s} \sim 5 a$.u. Therefore, we can approximate the vector $\mathbf{r}_{a b}=\mathbf{r}_{a}-\mathbf{r}_{b}$ in the argument of the interaction factor $F_{\text {int }}\left(\mathbf{p}_{12}, \mathbf{r}_{a b}\right)$ in Eq. (17) by the radius vector of the outer electron: $\mathbf{r}_{a b}=\mathbf{r}_{a}$ if the index $a$ corresponds to the $2 s$ electron and $\mathbf{r}_{a b}=-\mathbf{r}_{b}$ in the opposite case, $a=1 s$. Thus, we neglect the coordinates of the inner-electron in the interaction factor $F_{\text {int }}$ of the final wave function. This simplification can be also justified because the dependence of the interaction factor $F_{i n t}\left(\mathbf{p}_{12}, \mathbf{r}_{a b}\right)$ upon the inner electron coordinate on the scale of the inner orbit does not actually affect the correlation function. This feature of the interaction factor has been proven in our previous paper where we considered the ionization of the helium atom from the ground state. That work demonstrated that the spatial dependence of $F_{\text {int }}\left(\mathbf{p}_{12}, \mathbf{r}_{a b}\right)$ on the scale $r_{a b} \sim 1 a$ a. is not essential for the behavior of the correlation function $R$ and, thus, $r_{a b}$ can be put equal to zero if the ground state is considered. However, this is not valid for the excited 1s2s state whose outer radius $r_{2 s} \gg 1$ a.u. and the spatial dependence of $F_{\text {int }}\left(\mathbf{p}_{12}, \mathbf{r}_{a b}\right)$ upon the coordinates of the outer electron should be properly taken into account. This circumstance makes the evaluation of the ionization amplitude much more complicated than in the case of double ionization from the ground state. Note that in the latter case it was possible to derive an analytical closed expressions for the ionization amplitude (12) and differential cross section (11).

Nevertheless, even in the case of an excited target atom, the coordinates of the inner electron can be neglected in the argument of $F_{\text {int }}$, which will considerably simplify our further calculations. Indeed, it is easy to see that if we assume that $F_{\text {int }}$ depends on the coordinates of outer electrons only, the BBK wave function actually reduces to the product of the single electron wave functions and, therefore, the ionization amplitude $T_{f i}$ splits into the products of the uncoupled single particle matrix elements corresponding to the ionization of $1 \mathrm{~s}$ and $2 \mathrm{~s}$ electrons. Note that correlation is still present in amplitude through the dependence of the interaction factor $F_{\text {int }}$ upon the momentum difference $\Delta p$. There are two types of single particle matrix elements entering the expression for $T_{f i}$ : the overlap integrals of the final and initial wave functions corresponding to the shake-off amplitudes and the dipole matrix elements corresponding to the knock-out amplitudes. Thus, within the Born approximation, double ionization is represented as a knock-out process of the first target electron and a shake-off of the second.

The large difference between $r_{2 s}$ and $r_{1 s}$ radii leads to another simplification. In this case the probability for the shake-off ionization of the inner electron proves to be negligibly small. Indeed, the influence of the outer electron on the deep 1s electron state is not essential. Therefore, it is unlikely that the knock-out of the $2 \mathrm{~s}$ electron could result in shake-off ionization of the 1s electron. This fact can be seen from the properties of the electron wave functions. According to Table 1 , the $1 \mathrm{~s} \operatorname{HF}$ wave function $\varphi_{1 s}(\mathbf{r})$ is close to the simple hydrogenic 1 s wave function. Therefore, the shake-off amplitude for the 1 s state, i.e. the overlap integral of the 1 s and continuum wave functions $\left\langle\varphi_{\mathbf{p}} \mid \varphi_{1 s}\right\rangle$, is negligibly small because of orthogonality of the initial $\varphi_{1 s}$ and continuum wave functions 
$\varphi_{\mathbf{p}}$ which both correspond to the electron motion in the unique field of the doubly charged nucleus of the target. Neglecting this matrix element, we derive from Eqs. (12)-(18) that the ionization amplitude is equal to

$$
\begin{aligned}
T_{f i}=\frac{4 \pi Q}{q^{2}} & {\left[\left\langle\varphi_{\mathbf{p}_{1}}|\mathbf{q} \cdot \mathbf{r}| \varphi_{1 s}\right\rangle\left\langle\varphi_{\mathbf{p}_{2}}\left|F_{i n t}^{*}\left(\mathbf{p}_{21}, \mathbf{r}\right)\right| \varphi_{2 s}\right\rangle+\right.} \\
& \left.+(-1)^{S}\left\langle\varphi_{\mathbf{p}_{2}}|\mathbf{q} \cdot \mathbf{r}| \varphi_{1 s}\right\rangle\left\langle\varphi_{\mathbf{p}_{1}}\left|F_{\text {int }}^{*}\left(\mathbf{p}_{12}, \mathbf{r}\right)\right| \varphi_{2 s}\right\rangle\right],
\end{aligned}
$$

where $\mathbf{p}_{21}=-\mathbf{p}_{12}$ and knock-out and shake-off amplitudes are equal to

$$
\begin{aligned}
& \left\langle\varphi_{\mathbf{p}_{i}}|\mathbf{q} \cdot \mathbf{r}| \varphi_{1 s}\right\rangle=\int \varphi_{\mathbf{p}_{i}}^{*}(\mathbf{r})(\mathbf{q} \cdot \mathbf{r}) \varphi_{1 s}(\mathbf{r}) d \mathbf{r} \\
& \left\langle\varphi_{\mathbf{p}_{i}}\left|F_{i n t}^{*}\left(\mathbf{p}_{i j}, \mathbf{r}\right)\right| \varphi_{2 s}\right\rangle=\int \varphi_{\mathbf{p}_{i}}^{*}(\mathbf{r}) F_{i n t}^{*}\left(\mathbf{p}_{i j}, \mathbf{r}\right) \varphi_{2 s}(\mathbf{r}) d \mathbf{r}
\end{aligned}
$$

respectively. According to the expression (19) the double ionization of the helium atom in the process under study is considered as the knock-out of the 1s electron by ion impact followed by the shake-off of the 2 s electron which is influenced by both the field of a doubly charged ion and the field of an emitted electron. The two terms in the ionization amplitude (19) correspond to the exchange between the two escaping electrons with momenta $\mathbf{p}_{1}$ and $\mathbf{p}_{2}$.

The knock-out amplitude (20) can be evaluated analytically using the partial wave expansion of the Coulomb wave $\varphi_{\mathbf{p}_{i}}$ (Messiah 1970), resulting in:

$$
\begin{aligned}
& \left\langle\varphi_{\mathbf{p}_{i}}|\mathbf{q} \cdot \mathbf{r}| \varphi_{1 s}\right\rangle=-\frac{4 \sqrt{2} \mathrm{i}}{\pi} F_{\text {Som }}\left(\xi_{i}\right)\left(\mathbf{q} \cdot \mathbf{p}_{i}\right) A_{1}^{1 s}\left(p_{i}\right) \\
& A_{1}^{1 s}\left(p_{j}\right)=\sum_{i=1}^{2} B_{i}^{1 s}\left(1+\mathrm{i} \xi_{j}\right) \frac{\beta_{i}^{1 s}-1}{\left(\left(\beta_{i}^{1 s}\right)^{2}+p_{j}^{2}\right)^{3}} \exp \left[2 \xi_{j} \arctan \left(\frac{p_{j}}{\beta_{i}^{1 s}}\right)\right] .
\end{aligned}
$$

Evaluating the shake-off matrix element (21) we first perform analytically the integration over the modulus of the vector $\mathbf{r}$. The result of such an integration, the function $J\left(\mathbf{p}_{i}, \mathbf{p}_{i j}, \beta_{k}^{2 s}, \Omega_{\mathbf{r}}\right)$, is presented in the Appendix. Then, integration over the solid angle $\Omega_{\mathbf{r}}$ is carried out numerically:

$$
\begin{aligned}
& \left\langle\varphi_{\mathbf{p}_{i}}\left|F_{i n t}^{*}\left(\mathbf{p}_{i j}, \mathbf{r}\right)\right| \varphi_{2 s}\right\rangle=\frac{1}{4 \sqrt{2} \pi^{2}} F_{S o m}\left(\xi_{12}\right) F_{S o m}\left(\xi_{i}\right) A_{0}^{2 s}\left(\mathbf{p}_{i}, \mathbf{p}_{i j}\right) \\
& A_{0}^{2 s}\left(\mathbf{p}_{i}, \mathbf{p}_{i j}\right)=\sum_{k=1}^{3} B_{k}^{2 s} \int J\left(\mathbf{p}_{i}, \mathbf{p}_{i j}, \beta_{k}^{2 s}, \Omega_{\mathbf{r}}\right) d \Omega_{\mathbf{r}}
\end{aligned}
$$

Substituting the matrix elements (22) and (24) in the transition amplitude (19) and evaluating the integration in Eq. (11) over $d \mathbf{q}$ we obtain finally for the differential ionization cross section the following expression 


$$
\begin{aligned}
\frac{d^{6} \sigma}{d \mathbf{p}_{1} d \mathbf{p}_{2}}= & \frac{4}{\pi^{5}}\left(\frac{Q}{v}\right)^{2} \ln \left(\frac{q_{\text {max }}}{q_{\text {min }}}\right)\left|F_{\text {Som }}\left(\xi_{1}\right) F_{\text {Som }}\left(\xi_{2}\right) F_{\text {Som }}\left(\xi_{12}\right)\right|^{2} \times \\
& \times\left|A_{1}^{1 s}\left(p_{1}\right) A_{0}^{2 s}\left(\mathbf{p}_{2}, \mathbf{p}_{21}\right) \mathbf{p}_{\perp 1}+(-1)^{S} A_{1}^{1 s}\left(p_{2}\right) A_{0}^{2 s}\left(\mathbf{p}_{1}, \mathbf{p}_{12}\right) \mathbf{p}_{\perp 2}\right|^{2}
\end{aligned}
$$

Here $\mathbf{p}_{\perp 1,2}=\mathbf{p}_{1,2}-\mathbf{e}_{\mathbf{P}}\left(\mathbf{e}_{\mathbf{P}} \cdot \mathbf{p}_{1,2}\right)$ are the projections of the electron momentum vectors onto the plane perpendicular to the direction of the incoming ion, and the unit vector $\mathbf{e}_{\mathbf{P}}=\mathbf{P} / P$ is oriented along the direction of the incoming ion.

The minimum value of the transferred momentum, $q_{\text {min }}$, is given by Eq. (10). The maximum value, $q_{\max }$, is determined by the condition of validity of the dipole approximation and can be estimated from the inequality: $\left|\left\langle\varphi_{p}\left|\mathbf{q}_{\max } \cdot \mathbf{r}\right| \varphi_{1 s}\right\rangle\right| \lesssim$ $\left|\left\langle\varphi_{p} \mid \varphi_{1 s}\right\rangle\right|$. Our numerical calculations show that $\ln \left(q_{\max } / q_{\min }\right)$ does not strongly vary in the considered momentum range and only slightly changes the correlation function. Therefore, it can be replaced by a constant.

It should be noted that the expression (26) obtained for the differential cross section is determined by the sum of two amplitudes which differ by the exchange of the escaping electrons. Each amplitude is given by the product of the matrix elements describing the ejection of a 1s electron by momentum transfer from the projectile ion, i.e. by the knock-out process, and the ejection of a $2 \mathrm{~s}$ electron due to the change of the atomic field, i.e. by the shake-off process. The latter mechanism proves to be influenced by the Coulomb field of the first ejected electron. These factors determine the behavior of the correlation function $R$ and provide the main contribution to electron correlation in the double ionization process.

\section{Results and analysis}

We have calculated the correlation function $R$ for double ionization (1) of excited helium atoms in collisions with fast, $v=60 a . u$., carbon ions $C^{+6}$. We choose this projectile because it had been used before in experimental and theoretical studies of electron correlation in double ionization of the helium ground state (Bapat et al 1999, Bapat et al 2000, Keller et al 2000, Schulz et al 2000, Feuerstein et al 2001, Gerchikov and Sheinerman 2001). A rather high value of the projectile velocity $v=60 a . u$. and a small value of the Born parameter $Q / v=0.1$ justify the employed approximations for this collision system. The correlation function $R(4)$ has been calculated according to Eqs. (2)-(3) with the differential cross section given by Eq. (26). It is useful for better understanding of electron correlation in double ionization of excited helium also to compare the obtained results with the corresponding data for the helium ground state (Gerchikov and Sheinerman 2001).

In order to give more insight into the double ionization mechanism we have plotted in Fig. 1 the intensity $I_{\text {cor }}$ multiplied by the total cross section $\sigma_{\text {tot }}$ which corresponds to the differential cross section $d \sigma / d \Delta p=\sigma_{\text {tot }} I_{\text {cor }}$ (see Eqs. $(2,3,5)$ ). The solid line corresponds to the singlet, ${ }^{1} S$, initial state of the target while the dashed line shows 
$d \sigma / d \Delta p$ for ionization of the triplet state, ${ }^{3} S$. For comparison we also plot in another scale $d \sigma / d \Delta p$ for ionization of the ground state of the helium atom (dotted line). We plot $d \sigma / d \Delta p$ in arbitrary units because our approach does not yield correct absolute values of the cross sections (see Section 2).

Obviously, the value of the cross section is quite sensitive to the symmetry (or to the total spin $S$ ) of the target electrons. The total cross section $\sigma_{t o t}$ for double ionization of the triplet initial state is two times larger than for the singlet state. It results from the interference between two terms in the ionization amplitude $T_{f i}$ (19) (see also Eq. (26)) which differ by the exchange of escaping electrons. Note, that in spite of the big difference between the cross sections, the two interfering terms are not comparable. Actually, one of them is several times smaller than the other. Thus, the leading term is the same for both, triplet and singlet amplitudes, and, that is why the shapes of $I_{c o r}$ for triplet and single states are similar, though the interference with exchange term alters the magnitude of the cross section. The following qualitative consideration might explain why the triplet cross section is larger. The sign of the interference, whether it is constructive or deconstructive, depends upon the momentum vectors of two emitted electrons. The configuration of the electronic momenta that favors most effectively the correlated electron emission is governed by the following two tendencies. On the one hand the momentum difference $\Delta p$ should not be too small, otherwise electron emission will be suppressed by the Coulomb repulsion (see Fig. 1). On the other hand the knock-out and shake-off amplitudes for each ejected electron rapidly decreases for large momenta $p>1$ as a function of $p$. The competition of these two tendencies results in a preferable electron emission into opposite directions. For such a momentum configuration the interference according to Eq. (26) will favor the triplet state. Indeed, it is easy to see that, for example, for pure back-to-back emission with equal momentum the differential cross section equals to zero for the singlet state exactly.

Although the employed approach does not provide the correct absolute values of the ionization cross section, it allows us to compare their relative values. Obviously, the value of $d \sigma / d \Delta p$ for the excited state is much larger than for the ground target state due to the large radius of the $2 \mathrm{~s}$ orbit. Roughly speaking, the ionization cross section of the excited state and, consequently, the value of $d \sigma / d \Delta p$ increases as the square of the outer electron's orbit radius.

The overall behavior of all of the curves are quite similar apart from the observation that the maxima of $d \sigma / d \Delta p$ are shifted, most pronounced for the ground state where it is observed towards larger values of $\Delta p$. The main features, i.e. the strong maximum between $\Delta p \sim 1.5$ a.u. and $2 a . u$. and the rapid decrease of $d \sigma / d \Delta p$ at smaller and larger $\Delta p$ values can be easily understood by taking into account that the intensity of the electron emission is strongly suppressed for small $\Delta p(\Delta p<1$ a.u. $)$ by the Coulomb repulsion between escaping electrons with close momenta. In this region the dependence $d \sigma / d \Delta p$ is governed by the Sommerfeld factor (16). In the region $\Delta p>2.0 a . u$. the cross section decreases, vanishing at large $\Delta p$ because of the small probability of electron emission with a large momentum in the knock-out process ( small values of momentum 
transfer $q$ are essential in fast collisions) and in the shake-off process as well.

In Fig. 2 we present the correlation function $R(\Delta p)$ for the singlet (solid line) and triplet (dashed line) 1s2s states together with the correlation function for the ground state (dotted line). Fig. 2 shows that the overall shape of correlation functions are generally similar. The reasons for such a behavior are the same as for the differential cross section discussed above. Its detailed analyses has been done in our previous work (Gerchikov and Sheinerman 2001). In the present paper we will focus our attention on the distinct difference between the correlation functions corresponding to excited and ground initial states and less pronounced but still visible differences between $R$ for the exited states. The maximum of $R(\Delta p)$ for the excited states at $\Delta p \sim 1.8$ a.u. becomes higher and narrower and its position is shifted towards smaller values of $\Delta p$ compared to the ground state. Note that the correlation function was found to be rather insensitive to the details of the description of the ground state electron wave function. Gerchikov and Sheinerman (2001) investigated this by choosing of different approximations for the initial $1 s^{2}$ ground state wave function of helium. Having this in mind, the obtained difference between the $R$ functions of excited and ground state are considerable, exceeding the resolution of the existing experimental techniques (Schulz et al 2000) and, therefore, may be distinguished in future experiments.

It should be also noticed that the correlation function is less sensitive to the total spin $S$ of emitted electrons than $d \sigma / d \Delta p$. Spin determines the sign of the interference between the direct and exchange ionization amplitudes in Eqs. (19, 26) and, therefore, the difference between the singlet and triplet $R$ functions characterizes the role of this interference in the electron correlation. In the case of the ground state its contribution to the correlation function was considerable (Gerchikov and Sheinerman 2000). However, for the excited state this contribution is less important. This effect of "interference suppression" can be explained by the large difference between 1 s and 2 s electrons in comparison with identical 1s electrons of the ground state. Nevertheless, Fig. 2 demonstrates the clear difference between the singlet and triplet correlation functions in the region where $\Delta p$ is larger than the position of the maximum. Hence, the singlet and triplet $R$ functions may be distinguished in experiments with sufficiently small statistic errors.

As it has been found before, the most important contribution that can explain the behavior of the $R$ function in the case of an excited target is the Coulomb interaction between the emitted electrons. The significance of this contribution is connected with its role in the formation of the maximum in the $R(\Delta p)$ dependence. The strong Coulomb repulsion between escaping electrons with similar momenta leads to anticorrelated electron emission at small $\Delta p$. With the increase of $\Delta p$ the influence of this factor decreases which results in the sharp increase of $R(\Delta p)$ at the left shoulder of the maximum. The anticorrelation effect of the Coulomb repulsion is most effective in the range of $\Delta p$ where the kinetic energy of the relative motion of the two emitted electrons $\Delta p^{2} / 4$ is smaller than the potential energy of their Coulomb interaction $1 / r_{a b}$. Indeed, this region is forbidden for the classical motion and, therefore, the probability 
that ionization events occur here should be small. The most probable value of the initial separation $r_{a b}$ can be estimated by the averaged interelectronic distances in the target. Thus, our rough estimation $\Delta p \sim \sqrt{4 / r_{a b}}$ for the right edge of this region can be associated with the position of the maximum of the correlation function.

One can propose the following qualitative interpretation of this assumption. Let us consider the ionization process as a sudden transition from the bound to continuum states. The potential energy of the interelectronic repulsion $1 / r_{a b}$ at the instant of the actual ionization event corresponds to the most probable value of the emitted electron's kinetic energy in their center of mass frame $\Delta p^{2} / 4$. Consequently, the initial state could affect the correlation function by setting the initial separation $r_{a b}$ for the correlated motion of two electrons during the ionization. The increase of the excited state radius decreases the potential energy of the Coulomb repulsion at the instant of the ionization which in turn makes the left shoulder of the maximum sharper and narrower. Finally, it shifts the position of the maximum towards smaller values of $\Delta p$. Also one may note the small difference between the positions of maxima of the singlet and triplet correlation functions. It reflects the same tendency discussed above. The increase of the $2 \mathrm{~s}$ orbit radius for the ${ }^{1} S$ state compare to the ${ }^{3} S$ state shifts the position the maximum for the singlet $R$ function slightly to the left with respect to the triplet $R$ function. Though we have to note here that such a small momentum shift, less than 0.1 a.u., is close to the accuracy limit of the employed approximations.

In order to verify this qualitative explanation of the behavior of the $R$ function we performed the simulation calculations based on the approach developed in our earlier work (Gerchikov and Sheinerman 2001). We note that the interelectronic distance $r_{a b}$ which governs the Coulomb interaction of escaping electrons enters expressions for the correlation function via the argument of the $F_{\text {int }}\left(\mathbf{p}_{12}, \mathbf{r}_{a b}\right)$ factor in the BBK wave function (17). We replaced the actual vector $\mathbf{r}_{a b}$ in the expression (15) for $F_{\text {int }}$ by a parameter $\alpha$ which simulates the average separation of emitted electrons:

$$
F_{\text {int }}=F_{\text {Som }}\left(\xi_{12}\right) \cdot{ }_{1} F_{1}\left(\mathrm{i} \xi_{12}, 1,-\frac{\mathrm{i}}{2} p_{12} \alpha\right) \text {. }
$$

According to this definition, the factor $F_{\text {int }}$ which describes the interaction of ionized electrons is a function of the momentum difference only, $F_{\text {int }} \equiv F_{\text {int }}\left(p_{12}\right)$. Then, we calculated the correlation function $R$ (4) for different values of the parameter $\alpha$ using the new expression (27) for $F_{\text {int }}$ instead of Eq. (15) in the Eqs. (19-21) for ionization amplitudes. In Fig. 3 we present the results obtained for two values of the parameter $\alpha: \alpha=0$ (dash-dotted line) and $\alpha$ equal to the average electronic separation $\left\langle r_{a b}\right\rangle$ in the initial target state (dashed line). For the singlet state $\left\langle r_{a b}\right\rangle=5.16 a . u$. (Fig. 3a) and for the triplet state $\left\langle r_{a b}\right\rangle=4.56 a . u$. (Fig. 3b). For comparison we plot in Fig. 3a,b the exact correlation function (solid line) calculated according to Eqs. (2-3) with the differential cross section given by Eq.(26). In Fig 3a we also show by the dotted line the correlation function calculated for the ground state using the approximate Eq. (27) with parameter $\alpha=\left\langle r_{a b}\right\rangle=1.5$ a.u. (Gerchikov and Sheinerman 2001). We do not compare the ground state and excited ${ }^{3} S$ state correlation functions in Fig. $3 \mathrm{~b}$ because due to the 
different symmetry of the singlet and triplet states the interference between direct and exchange ionization amplitudes has a different character and as such the comparison is less informative than in Fig. 3a. Note here, that in the case of the ground state the variation of the parameter $\alpha$ in the interval $0<\alpha \lesssim 1.5$ a.u. does not actually change the correlation function. It means that the approximation (27) provides the proper accuracy for calculations in the case of the initial ground state. It should be noted, that if one uses the value of parameter $\alpha=0$ then an interelectron interaction is taken into account by the Sommerfeld factor $F_{\text {Som }}\left(\xi_{12}\right)$ solely. Such an approximation has been used in a number of sudies of double ionization of ground state targets (Maulbetsch and Briggs 1993, 1994, Hda et al 1994, Dal Capello et al 1995, Lamy et al 1996, Keller et al 1997, 2000, Sheinerman and Schmidt 1999, Schulz et al 2000).

Fig. 3 demonstrates that the main factor which determines the behavior of the correlation function is the initial separation between the escaping electrons. Indeed, in spite of the difference between the shapes of $2 \mathrm{~s}$ and $1 \mathrm{~s}$ wave functions the dotted and dash-dotted curves corresponding to $1 \mathrm{~s}^{2}$ and $1 \mathrm{~s} 2 \mathrm{~s}$ singlet initial states with similar $\alpha$ parameters are very close (see Fig. 3a). On the other hand increase of the initial electron separation in the $1 \mathrm{~s} 2 \mathrm{~s}$ state simulated by the parameter $\alpha=\left\langle r_{a b}\right\rangle$ (dashed line) changes the shape of the correlation function and brings it closer to the results of the exact calculations (solid line). It proves our conclusion that the Coulomb repulsion between ionized electrons together with their initial separation explains the alteration of the correlation function due to the increase of the initial excited state radius. It should be noticed that though the approximate (27) reproduces the main trends of the evolution of the correlation function it can not provide the high accuracy of the calculations for the initial excited state especially in the singlet case and more precise calculations using Eq. (26) are necessary.

\section{Conclusion}

A simple quantum mechanical approach within the first Born and dipole approximation has been developed to calculate the correlation function for double ionization of the excited helium atom. In such an approach the double ionization is dominated by the knock-out of the inner electron by the projectile ion and the shake-off of the outer electron. The electron correlation taken into account in our approach includes the electron interaction of the final state and the exchange process between the knock-out and shake-off electrons. We demonstrate that the correlation function in the case of the excited target differs from the correlation function for ground state ionization. The main factors which transform the correlation function pattern are the Coulomb repulsion between escaping electrons and the increase of the excited state radius. The initial state affects the correlation function by defining the initial separation for the correlated motion of the two electrons during the ionization. The reported dependence of the correlation function upon the radius of the excited state can be verified experimentally since the alteration of the correlation function exceeds the existing resolution of intensity 
interferometry methods. If our theoretical predictions can be verified experimentally it will open the opportunity to apply intensity interferometry methods for diagnostics of atomic and molecular targets.

\section{Acknowledgments}

It is a pleasure to thank Dr. A. Voitkiv for the fruitful discussion and Dr. J. Lambourne for careful reading of the manuscript. Support from the Leibniz-Programm of the DFG and by BMBF (06HD994) is gradually acknowledged.

\section{Appendix}

The radial part of matrix element (21) is defined as

$$
\begin{aligned}
J\left(\mathbf{p}_{i}, \mathbf{p}_{i j}, \beta_{k}, \Omega_{\mathbf{r}}\right)=\int d r r^{2} \exp \left(-\beta_{k} r+\mathrm{i} \mathbf{p}_{i} \mathbf{r}\right) \times \\
\times{ }_{1} F_{1}\left(\frac{2 \mathrm{i}}{p_{i}}, 1, \mathrm{i}\left(p_{i} r+\mathbf{p}_{i} \mathbf{r}\right)\right) \cdot{ }_{1} F_{1}\left(-\frac{\mathrm{i}}{p_{12}}, 1, \frac{\mathrm{i}}{2}\left(p_{i j} r+\mathbf{p}_{i j} \mathbf{r}\right)\right) .
\end{aligned}
$$

We will employ the following notations:

$$
\begin{aligned}
& \lambda_{k}=\beta_{k}+\mathrm{i} \mathbf{p}_{i} \mathbf{e}_{r} ; \quad d_{1}=\mathrm{i}\left(p_{i}+\mathbf{p}_{i} \mathbf{e}_{r}\right) ; \quad d_{2}=\frac{\mathrm{i}}{2}\left(p_{12}+\mathbf{p}_{i j} \mathbf{e}_{r}\right) ; \\
& a=\frac{2 \mathrm{i}}{p_{i}} \quad b=-\frac{\mathrm{i}}{p_{12}} ; \quad Z_{k}=\frac{d_{1} d_{2}}{\left(\lambda_{k}-d_{1}\right)\left(\lambda_{k}-d_{2}\right)} .
\end{aligned}
$$

Using recurrence relations (see Bateman and Erdelyi 1953) we express each of the confluent hypergeometric function in the Eq.(A.1) as a linear combination of three confluent hypergeometric functions with the second parameter equal to 3 . Then the integrand in the Eq.(A.1) includes the sum of nine terms that can be developed using the Nordsieck relation (Nordsieck 1954, see also Landau and Lifshitz 1977):

$$
\begin{gathered}
J=\int_{0}^{\infty} \exp (-\lambda z) z^{\gamma-1}{ }_{1} F_{1}(\alpha, \gamma, k z){ }_{1} F_{1}\left(\alpha^{\prime}, \gamma, k^{\prime} z\right) d z= \\
=\Gamma(\gamma) \lambda^{\alpha+\alpha^{\prime}-\gamma}(\lambda-k)^{-\alpha}\left(\lambda-k^{\prime}\right)^{-\alpha^{\prime}}{ }_{2} F_{1}\left(\alpha, \alpha^{\prime}, \gamma, \frac{k k^{\prime}}{(\lambda-k)\left(\lambda-k^{\prime}\right)}\right)
\end{gathered}
$$

with $\gamma=3$. The obtained hypergeometric functions can be transformed using the Gauss relations for adjacent functions (Bateman and Erdelyi 1953). As a result we have obtained the linear combination of the two hypergeometric functions:

$$
J\left(\mathbf{p}_{i}, \mathbf{p}_{i j}, \beta_{k}, \Omega_{\mathbf{r}}\right)=x_{1} \cdot{ }_{2} F_{1}\left(a, b+1,3, Z_{i}\right)+x_{2} \cdot{ }_{2} F_{1}\left(a+1, b, 3, Z_{i}\right) .
$$

The coefficients of this linear combination are expressed by a number of recurrence relations: 


$$
\begin{aligned}
& x_{1}=t_{1} \frac{2-a}{(b-a)\left(1-Z_{i}\right)}+t_{3}+t_{4} \frac{b}{b-a} ; \\
& x_{1}=t_{2}-t_{4} \frac{a}{b-a}-t_{1} \frac{2-b}{(b-a)\left(1-Z_{i}\right)} \text {; } \\
& t_{1}=s_{3}+s_{1} \frac{1-2 b+(b-a) Z_{i}}{(1+b)\left(Z_{i}-1\right)} ; \quad t_{2}=s_{5}+s_{1} \frac{b-2}{(1+b)\left(Z_{i}-1\right)} \\
& t_{3}=s_{4}+s_{2} \frac{1-2 b+(b-a+1) Z_{i}}{(1+b)\left(Z_{i}-1\right)} ; \quad t_{4}=s_{6}+s_{2} \frac{b-2}{(1+b)\left(Z_{i}-1\right)} \\
& s_{1}=r_{4}+r_{1} \frac{1-2 a+(a-b-1) Z_{i}}{(1+a)\left(Z_{i}-1\right)} ; \quad s_{2}=r_{7}+r_{1} \frac{a-2}{(1+a)\left(Z_{i}-1\right)} ; \\
& s_{3}=r_{5}+r_{2} \frac{1-2 a+(a-b) Z_{i}}{(1+a)\left(Z_{i}-1\right)} ; \quad s_{2}=r_{8}+r_{2} \frac{a-2}{(1+a)\left(Z_{i}-1\right)} ; \\
& s_{5}=r_{6}+r_{3} \frac{1-2 a+(a-b+1) Z_{i}}{(1+a)\left(Z_{i}-1\right)} ; \quad s_{6}=r_{9}+r_{3} \frac{a-2}{(1+a)\left(Z_{i}-1\right)} ; \\
& r_{1}=\frac{(a+1) a(b+1) b}{2} \lambda_{i}^{a+b+1}\left(\lambda_{i}-d_{1}\right)^{-a-2}\left(\lambda_{i}-d_{2}\right)^{-b-2} ; \\
& r_{2}=-(a+1) a b(b-1) \lambda_{i}^{a+b}\left(\lambda_{i}-d_{1}\right)^{-a-2}\left(\lambda_{i}-d_{2}\right)^{-b-1} \text {; } \\
& r_{3}=\frac{(a+1) a(b-1)(b-2)}{2} \lambda_{i}^{a+b-1}\left(\lambda_{i}-d_{1}\right)^{-a-2}\left(\lambda_{i}-d_{2}\right)^{-b} ; \\
& r_{4}=-a(a-1)(b+1) b \lambda_{i}^{a+b}\left(\lambda_{i}-d_{1}\right)^{-a-1}\left(\lambda_{i}-d_{2}\right)^{-b-2} ; \\
& r_{5}=2 a(a-1) b(b-1) \lambda_{i}^{a+b-1}\left(\lambda_{i}-d_{1}\right)^{-a-1}\left(\lambda_{i}-d_{2}\right)^{-b-1} ; \\
& r_{6}=-a(a-1)(b-1)(b-2) \lambda_{i}^{a+b-2}\left(\lambda_{i}-d_{1}\right)^{-a-1}\left(\lambda_{i}-d_{2}\right)^{-b} \text {; } \\
& r_{7}=\frac{(a-1)(a-2)(b+1) b}{2} \lambda_{i}^{a+b-1}\left(\lambda_{i}-d_{1}\right)^{-a}\left(\lambda_{i}-d_{2}\right)^{-b-2} ; \\
& r_{8}=-(a-1)(a-2) b(b-1) \lambda_{i}^{a+b-2}\left(\lambda_{i}-d_{1}\right)^{-a}\left(\lambda_{i}-d_{2}\right)^{-b-1} \text {; } \\
& r_{9}=\frac{(a-1)(a-2)(b-1)(b-2)}{2} \lambda_{i}^{a+b-3}\left(\lambda_{i}-d_{1}\right)^{-a}\left(\lambda_{i}-d_{2}\right)^{-b} .
\end{aligned}
$$




\section{References}

Bapat B, Keller S, Moshammer R, Mann R, and Ullrich J 2000 J. Phys. B: At. Mol. Opt. Phys. 33 $1437-50$

Bapat B, Moshammer R, Keller S, Schmitt W, Cassimi A, Adoui L, Kollmus H, Dörner R, Weber Th, Khayyat K, Mann R, Grandin J P and Ullrich J 1999 J. Phys. B: At. Mol. Opt. Phys. 32 1859-72

Bateman H and Erdelyi A 1953 Higher Transcendental Functions vol I,II (New York: Mc Graw-Hill)

Berakdar J and Klar H 1993 J. Phys. B: At. Mol. Opt. Phys. 26 4219-35

Brauner M, Briggs J S and Klar H 1989 J. Phys. B: At. Mol. Opt. Phys. 22 2265-87

Briggs J S and Schmidt V 2000 J. Phys. B: At. Mol. Opt. Phys. 33 R1-R48

Boal D H, Gelbke C K and Jennings B K 1990 Rev.Mod.Phys. 62553

Dal Capello C, Hda H and Roy A C 1995 Phys. Rev. A 515

Dörner R, Mergel V, Jagutzki O, Speilberger L, Ullrich J, Moshammer R, Schmidt-Böcking H 2000 Physics Reports 330 95-192

Feuerstein B, Schulz M, Moshammer R and Ullrich J 2001 Physica Scripta. T62 447-50

Garibotti G and Miraglia J E 1980 Phys. Rev. A 21572

Gerchikov L G and Sheinerman S A 2001 J. Phys. B: At. Mol. Opt. Phys. 34 647-57

Hda H, Dal Cappello C and Langlois J 1994 Z. Phys. D 2925

Keller S, Lüdde H J and Dreizler R M 1997 Phys. Rev. A 55 4215-20

Keller S, Bapat B, Moshammer R, Ullrich J and Dreizler R M 2000 J. Phys. B: At. Mol. Opt. Phys. 33 1447-61

Kheifets A S, Ipatov A, Arifin M and Bray I 2000 Phys. Rev. A 62052724

Lamy P, Joulakian B, Dal Cappello C and Lahmam- Bennani A 1996 J. Phys. B: At. Mol. Opt. Phys. $292315-23$

Landau L D and Lifschitz E M 1977 Quantum Mechanics (New York: Pergamon)

Lucey S P, Rasch J, Colm T Whelan and Walters H R J 1998 J. Phys. B: At. Mol. Opt. Phys. 31 $1237-58$

Maulbetsch F and Briggs J S 1993 J. Phys. B: At. Mol. Opt. Phys. 261679

1994 J. Phys. B: At. Mol. Opt. Phys. 274095

McGuire J H 1997 Electron Correlation Dynamics in Atomic Collisions, (Cambridge University Press)

Messiah A M L 1970 Quantum Mechanics, (New York: Wiley)

Nordsieck A 1954 Phys. Rev. 93785

Schulz M, Moshammer R, Schmitt W, Kollmus H, Feuerstein B, Mann R, Hagmann S and Ullrich J 2000 Phys. Rev. Lett. 84 863-6

Schulz M, Moshammer R, Gerchikov L G, Sheinerman S A and Ullrich J 2001 J. Phys. B: At. Mol. Opt. Phys. 34 L795-801

Sheinerman S A and Schmidt V 1999 J. Phys. B: At. Mol. Opt. Phys. 32 5205-20 


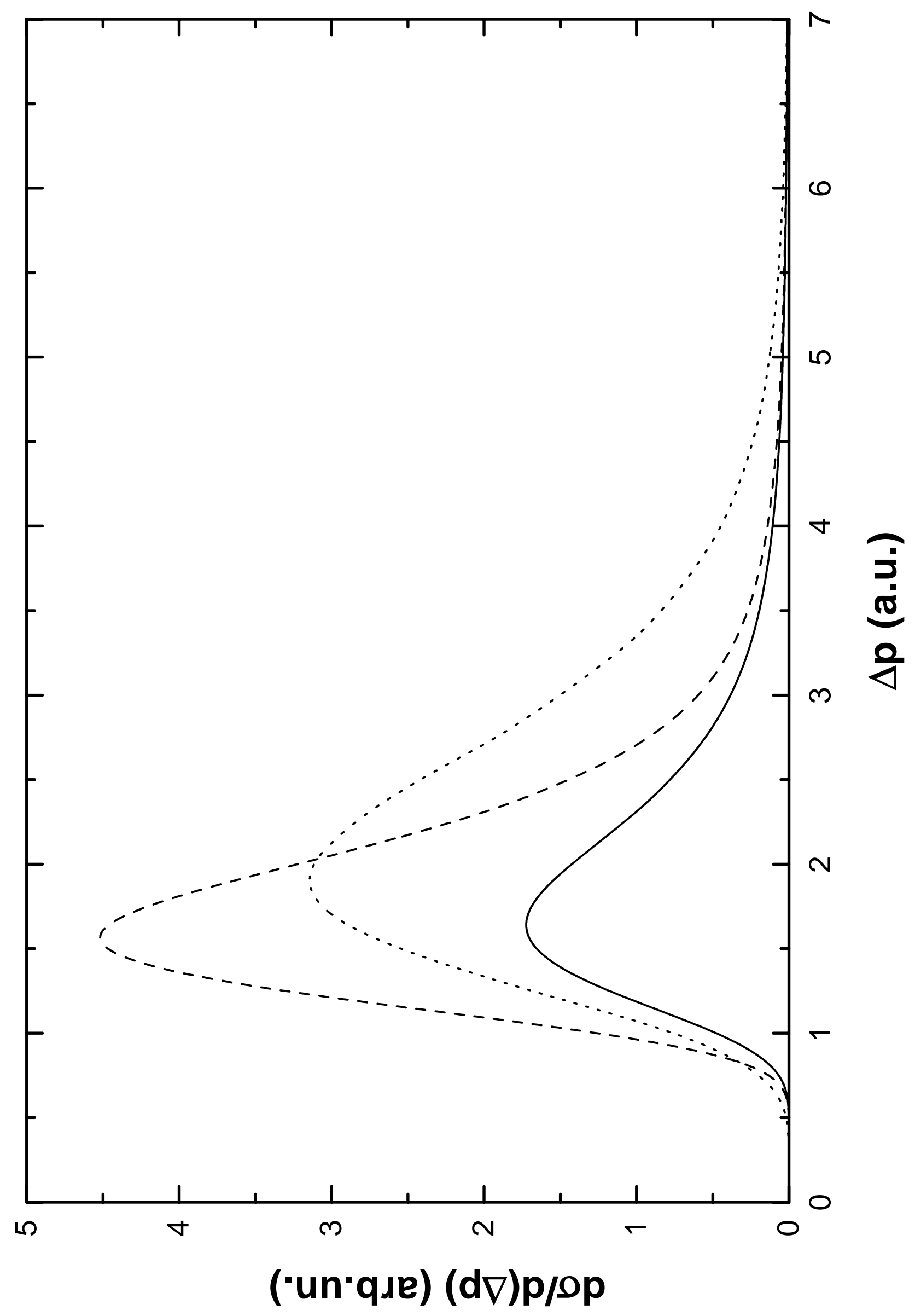




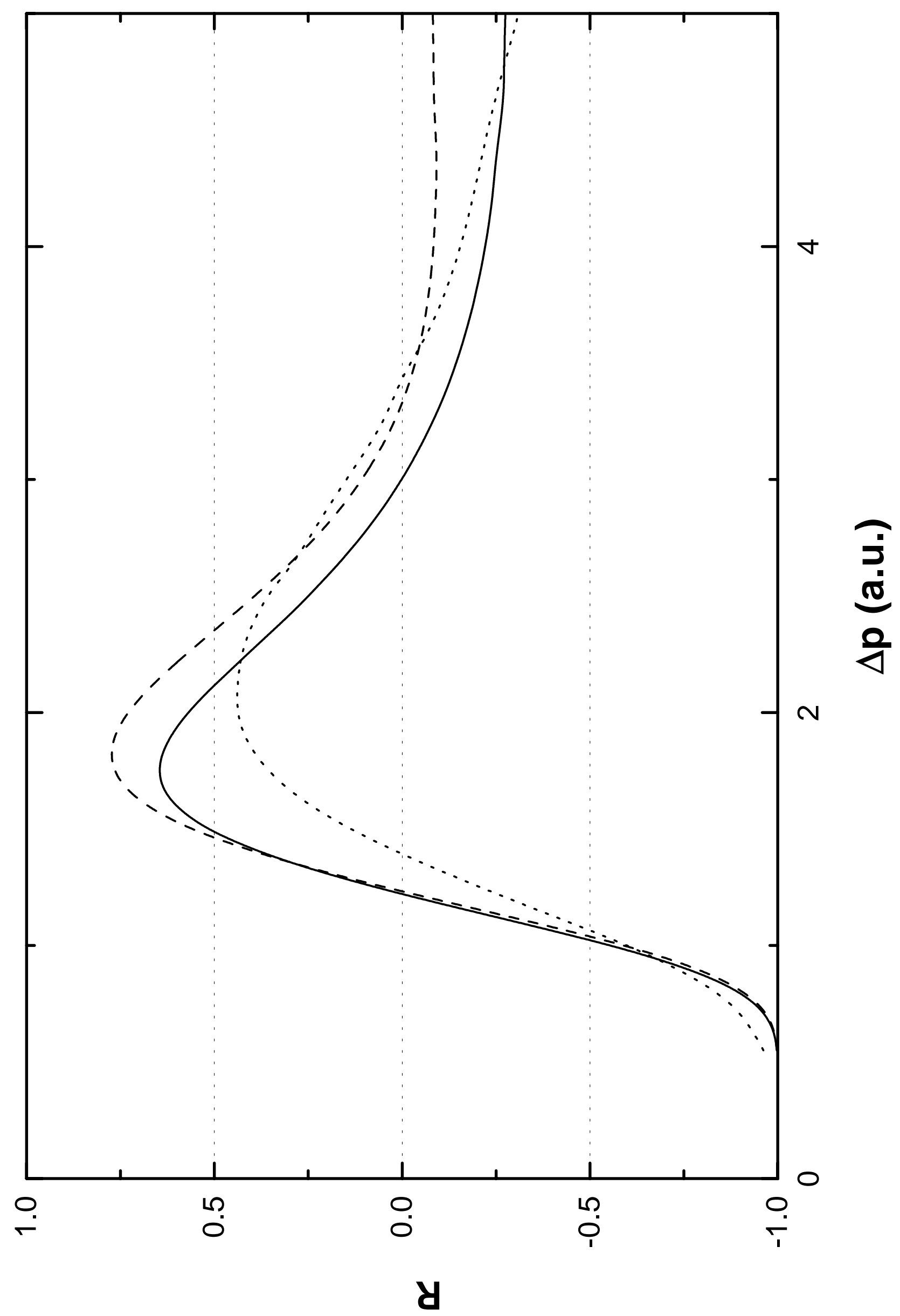




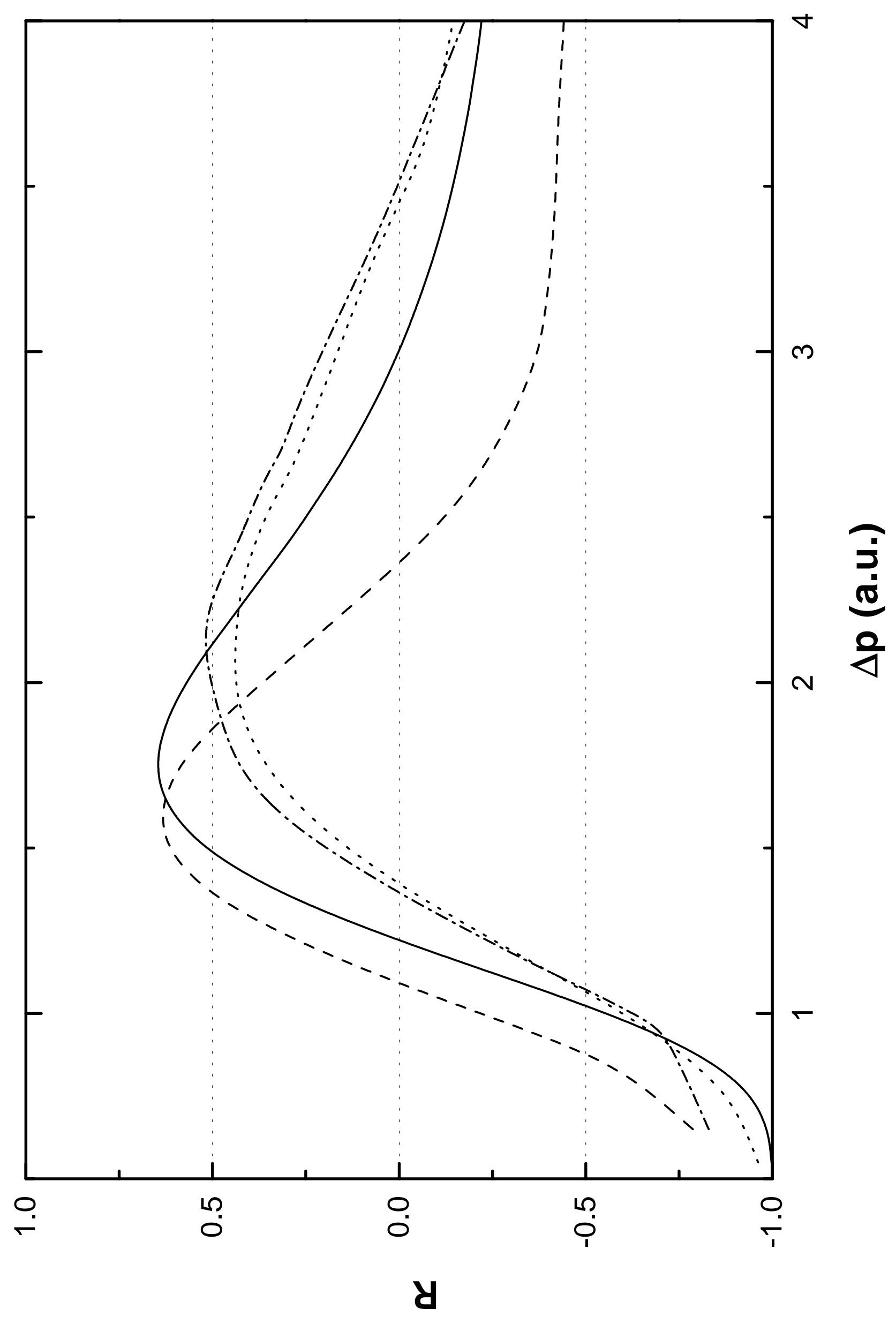




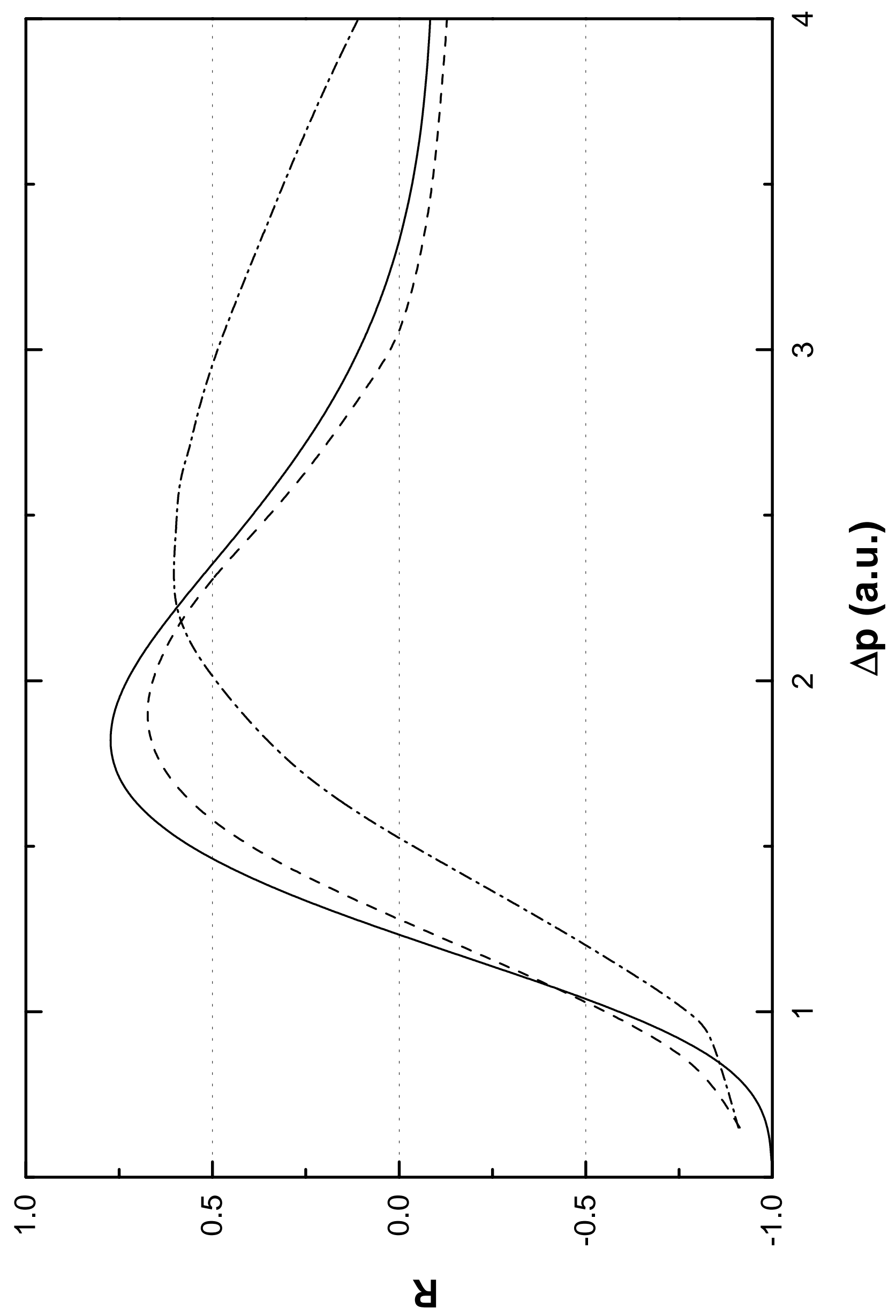




\section{Figure Captions}

Fig. 1: Differential double ionization cross section $d \sigma / d \Delta p$ as a function of momentumdifference for $100 \mathrm{MeV} / \mathrm{amu} \mathrm{C}^{6+}+\mathrm{He}(1 s 2 s)$ collisions. Solid and dashed lines correspond to the singlet and triplet initial states of excited $\mathrm{He}$, respectively. The dotted line shows $d \sigma / d \Delta p$ times 50 for the initial ground state of $H e$ calculated in (Gerchikov and Sheinerman 2001).

Fig. 2: The correlation function $R$ for $100 \mathrm{MeV} / \mathrm{amu} C^{6+}+H e(1 s 2 s)$ collisions calculated for the singlet (solid line) and triplet (dashed line) initial states of excited $H e$. The dotted line shows $R$ for the initial ground state of $H e$ calculated in (Gerchikov and Sheinerman 2001).

Fig.3: The correlation function $R$ for $100 \mathrm{MeV} / \mathrm{amu} C^{6+}+H e(1 s 2 s)$ obtained using the approximate $(27)$ for the two values of parameter $\alpha=0$ (dash-dotted line) and $\alpha=\left\langle r_{a b}\right\rangle$ (dashed line). The data for the singlet, $1 s 2 s\left({ }^{1} S\right)$, and triplet, $1 s 2 s\left({ }^{3} S\right)$, excited states are presented in Fig. 3a and Fig. 3b respectively. For the singlet state the initial electron separation is $\left\langle r_{a b}\right\rangle=5.16 a$.u. and for the triplet state $\left\langle r_{a b}\right\rangle=4.56$ a.u.. Solid lines represent the exact correlation function calculated according to Eqs. (2)-(3) with the differential cross section given by Eq.(26). In Fig. 3a we also show the correlation function calculated for the ground state (dotted line) using the approximate (27) with parameter $\alpha=\left\langle r_{a b}\right\rangle=1.5 a . u$. (Gerchikov and Sheinerman 2001).

\section{Table Captions}

Table 1 Parameters $B_{i}$ and $\beta_{i}$ for the approximation (14) of the single particle $\operatorname{HF} \varphi_{1 s}$ and $\varphi_{2 s}$ wave functions. 
Table 1

\begin{tabular}{|c|c|c|c|c|}
\hline & \multicolumn{2}{|c|}{${ }^{1} S$} & \multicolumn{2}{c|}{${ }^{3} S$} \\
\hline & $1 s$ & $2 s$ & $1 s$ & $2 s$ \\
\hline$B_{1}$ & 5.515 & 2.072 & 5.674 & 2.379 \\
\hline$\beta_{1}$ & 1.976 & 0.778 & 2.004 & 0.774 \\
\hline$B_{2}$ & 0.100 & -1.3686 & -0.009 & -1.984 \\
\hline$\beta_{2}$ & 3.360 & 0.4678 & 5.104 & 0.538 \\
\hline$B_{3}$ & - & 0.507 & - & 0.655 \\
\hline$\beta_{3}$ & - & 2.868 & - & 2.0437 \\
\hline
\end{tabular}

